\title{
A Multi-Scale Model of Soft Imperfect Interface with Nonlocal Damage
}

\author{
Asghar Ali Maitlo*, Frédéric Lebon ${ }^{\dagger}$ and Caroline Bauzet ${ }^{*}$ \\ Aix-Marseille University, CNRS \\ Centrale Marseille, LMA \\ Marseille France \\ *maitlo@lma.cnrs-mrs.fr \\ †lebon@lma.cnrs-mrs.fr \\ \$bauzet@lma.cnrs-mrs.fr
}

\begin{abstract}
The aim of this paper is to propose a model of bonded interface including nonlocal damage and unilateral conditions. The model is derived from the problem of a composite structure made by two adherents and a thin adhesive. The adhesive is damaged at microscopic level and is subjected to two regimes, one in traction and one in compression. The model of interface is derived by matched asymptotic expansions. In this paper, two cases corresponding to the two regimes are discussed. Moreover, this model can be considered as a model of contact with adhesion and unilateral constraint. At the end of the paper, a simple numerical example is presented to show the evolution of the model.
\end{abstract}

Keywords: Matched asymptotic expansions; nonlocal damage; soft imperfect interface.

\section{Introduction}

In recent years, scientists and engineers have been interested in the structural assembly of materials, giving rise to the study of interfaces, in particular in presence of damage between matrix and fibers. It is very important to control and to predict the damage in the interface in order to propose models which are useful to design robust and stable structures. Moreover, adhesive bonding technology is now widely employed in engineering structural assembly and especially in aeronautics industry, where the use of composite materials is necessary to lighten structures. For further research on stratified composite materials and interface modeling, one can refer to, e.g., Refs. 1-15. Due to the presence of the adhesive layer, adhesive bonding joints are subjected to a complex state of stress with high stress concentrations and, consequently, accurate analysis and modeling of adhesive materials and bonded joints are 
required. Because the adhesive layer is often soft and usually very thin when compared with the characteristic dimensions of the structure, a relatively large number of elements in the thickness direction is necessary to achieve sufficiently accurate calculations in standard existing finite element codes. This gives rise to a large number of degrees of freedom and high simulation costs. To successfully deal with this difficulty, interphase's modeling has to precede the computation of the numerical solution. A classical modeling approach consists in describing the adhesive as a distinct lower-dimensional continuum, i.e., a surface material. To obtain this surface material, a classical methodology consists in the study of the convergence towards zero of one or more small parameters (the thickness of the bond, its mechanical characteristics, etc.) in the PDEs describing the equilibrium of the structure. Furthermore, when the adhesive layer is cracked at the micro scale, it is possible to take into account in the model as a damage parameter the local average length of the cracks. The procedure adopted in this paper is a generalization of the study proposed in Bonetti et al. ${ }^{16}$ In our analysis, two models of damaged composite structures have been introduced. The first model, which is classical, is based on the study of two adherents and an adhesive which is cracked at the microscopic level. The second model which is an imperfect interface model has been derived by asymptotic analysis from the first one. Moreover, the asymptotic analysis gives some justification to adhesive contact models studied in Bonetti et al. ${ }^{17,18}$ In this paper, we are investigating the damage in an adhesive at microscopic level, which allows the use of Phase field theory. Therefore, in the light of literature the crack length given by Bonetti et al. ${ }^{16}$ can be interpreted as a damage parameter and its limit as an intensity of adhesion parameter Bonetti et al. ${ }^{17,18}$ In this paper, a more general approach is proposed. The idea is to put forward a general methodology able to derive, in the nonlocal case, adhesion models.

In this paper, a model of damaged isotropic composite materials with nonlocal damage is studied. In this model, two adherents and an adhesive are considered. The adhesive which is cracked at microscopic level is subjected by two regimes, one in traction, in which the material is considered soft, and one in compression, in which the bulk parameter is taken as hard. Moreover, the damage in adhesive is evolutive and nonlocal. The interface model is derived from the first one by using the Matched Asymptotic Expansions method (see Refs. 15, 19-25). A model of soft imperfect interface (the interphase is replaced by an interface) with nonlocal damage and unilateral conditions is obtained.

This paper is divided in three sections, in the first section, the damage model for a composite structure is presented which includes two adherents and one adhesive. In the second section, the asymptotic method is applied to derive the model of imperfect interface. Furthermore, in the imperfect interface model, the damage effect is obtained as nonlocal. It is also obtained that there is no penetration between adherents. At the end of the paper, a simple one dimensional example is proposed to show the behavior of the model. 


\section{The Mechanical Model}

We consider a composite body made of three different materials, two elastic materials which are known as adherents and the third one which is the adhesive and has a nonlinear behavior. The adhesive is very thin in dimension as compared to the adherents, which posses thickness $\varepsilon>0$, and is also called interphase. The body occupies the total bounded smooth domain $\Omega^{\varepsilon} \in \mathbb{R}^{3}$ and is assumed to be perfectly bonded. We introduce an orthogonal frame of reference $\left(O, e_{1}, e_{2}, e_{3}\right)$. Let $\left(x_{1}, x_{2}, x_{3}\right)$ denote the position of a particle in the three dimensional frame. The center of the interphase mid-plane denotes the origin of the frame of reference and $x_{3}$-axis runs perpendicular to the open bounded set $S$, where $S=\left\{\left(x_{1}, x_{2}, x_{3}\right) \in \Omega^{\varepsilon} ; x_{3}=0\right\}$ as shown in Fig. 1 (left).

The domains of the adhesive, adherents, interfaces between adhesive and adherents, respectively, are given below.

$$
\begin{aligned}
& B^{\varepsilon}=\left\{\left(x_{1}, x_{2}, x_{3}\right) \in \Omega^{\varepsilon}\left|x_{3}\right|<\frac{\varepsilon}{2}\right\}, \\
& \Omega_{ \pm}^{\varepsilon}=\left\{\left(x_{1}, x_{2}, x_{3}\right) \in \Omega^{\varepsilon} \pm x_{3}>\frac{\varepsilon}{2}\right\}, \\
& S_{ \pm}^{\varepsilon}=\left\{\left(x_{1}, x_{2}, x_{3}\right) \in \Omega^{\varepsilon} x_{3}= \pm \frac{\varepsilon}{2}\right\} .
\end{aligned}
$$

We apply some external force $g$ on some part of surface boundary $\partial \Omega^{\varepsilon}$ of the domain $\Omega^{\varepsilon}$ which is denoted by $S_{g}$. One another part of the surface boundary $\partial \Omega^{\varepsilon}$ is denoted by $S_{u}$. We consider that $S_{u}$ has a strictly positive measure and a zero displacement field. Moreover, both parts of surface boundary are disjoint, i.e., $S_{g} \cap S_{u}=\emptyset$. Further, it is also assumed that both parts, i.e., $S_{g}$ and $S_{u}$, are located far away from the adhesive or interphase. It is considered that body force $f$ is acting on $\Omega^{\varepsilon} \backslash B^{\varepsilon}$ only. Moreover $u^{\varepsilon}$ and $\sigma^{\varepsilon}$ denotes, respectively the displacement field and the Cauchy stress tensor field. Under the small deformation assumption the strain tensor can be written as $e_{i j}=\frac{1}{2}\left(u_{i, j}^{\varepsilon}+u_{j, i}^{\varepsilon}\right)$, where "," denotes partial derivative.

As we took adherents as elastic then the stress tensor can be written as

$$
\sigma_{i j}^{\varepsilon}=a_{i j h k}^{ \pm} e_{h k}\left(u^{\varepsilon}\right)
$$

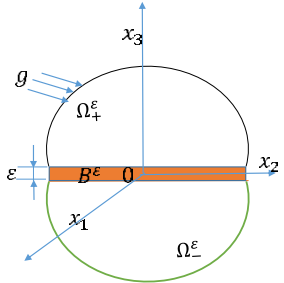

(a)

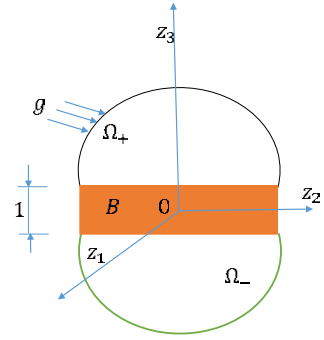

(b)

Fig. 1. Assembled composite structure. Left: Initial. Right: Rescaled. 
which is taken from the classical constitutive equation

$$
\sigma^{\varepsilon}=\psi_{, e}^{ \pm}\left(e\left(u^{\varepsilon}\right)\right)
$$

where $\psi^{ \pm}=\frac{1}{2} a^{ \pm} e\left(u^{\varepsilon}\right): e\left(u^{\varepsilon}\right)$ denotes the free energy, and $a^{ \pm}$is the fourth-order tensor of elasticity, which satisfies both classical positivity and symmetry conditions.

It is assumed in the following that the adhesive is damaged, i.e., its mechanical parameters depend on a damage parameter noted $\chi$ with $\chi \in[0,1]$. The case $\chi=1$ corresponds to a safe material and the case $\chi=0$ to a totally damaged material. For example, using the Kachanov theory et al. ${ }^{26,27,13}$ the damage parameter $\chi$ can be taken as $O\left(1 / l^{2}\right)$, where $l$ is an averaged crack length. In addition, the material is assumed to be isotropic. Therefore, in three dimension Hooke's law can be written as

$$
\sigma=\lambda(\chi) \operatorname{tr}(e) I_{2}+2 \mu(\chi)(e),
$$

where $I_{2}$ is the second-order identity tensor, $\lambda, \mu$ are the Lamé coefficients and "tr" indicates trace of a second-order tensor.

In addition, the material has two regimes, one in traction, one in compression. In traction, $\lambda$ and $\mu$ depend on the adhesive thickness and in compression we assume that only $\mu$ depends on the thickness. For traction, we take $\lambda=\varepsilon \lambda^{0}, \mu=\varepsilon \mu^{0}$ and for compression $\lambda=\lambda^{1}, \mu=\varepsilon \mu^{0}$. Then, the two regimes in the adhesive are defined by

$$
\begin{cases}\sigma^{\varepsilon}=\varepsilon \lambda^{0}(\chi) \operatorname{tr} e\left(u^{\varepsilon}\right) I_{2}+2 \varepsilon \mu^{0}(\chi) e\left(u^{\varepsilon}\right) & \text { if } \operatorname{tr} e\left(u^{\varepsilon}\right) \geq 0, \\ \sigma^{\varepsilon}=\lambda^{1}(\chi) \operatorname{tr} e\left(u^{\varepsilon}\right) I_{2}+2 \varepsilon \mu^{0}(\chi) e\left(u^{\varepsilon}\right) & \text { if } \operatorname{tr} e\left(u^{\varepsilon}\right) \leq 0 .\end{cases}
$$

As in Frémond ${ }^{28}$ a pseudo-potential of dissipation $\phi$ is introduced. In this paper, we suppose the following form for $\phi$ :

$$
\phi(\dot{\chi})=\frac{1}{2} \eta^{\varepsilon} \dot{\chi}^{2}+I_{[-\infty, 0]}(\dot{\chi}),
$$

where $\eta^{\varepsilon} \geq 0$ is a viscosity parameter and $I_{A}$ denotes the indicator function of the set $A$, which says that $I_{A}(x)=0$ if $x \in A$ and $I(x)=+\infty$ otherwise. The aim of the indicator function $I$ is to ensure that $\dot{\chi}$ must be negative, which gives the irreversible evolution of damage, i.e., the damage parameter is monotonically decreasing from 1 to 0 . Note that the material is chosen as rate-dependent. ${ }^{16}$

The free energy related to the constitutive Eq. (4) is divided into two parts, one concerning the strain and the other concerning the damage and is defined by

$$
\begin{aligned}
& \varepsilon\left(e\left(u^{\varepsilon}\right), \chi\right)=\bar{\psi}^{\varepsilon}\left(e\left(u^{\varepsilon}\right), \chi\right)+\tilde{\psi}^{\varepsilon}(\chi), \\
& \bar{\psi}^{\varepsilon}\left(e\left(u^{\varepsilon}\right), \chi\right)=\frac{1}{2}\left[\lambda(\chi) \operatorname{tr} e\left(u^{\varepsilon}\right)^{2}+2 \mu(\chi) e\left(u^{\varepsilon}\right)^{2}\right], \\
& \tilde{\psi}^{\varepsilon}(\chi)=-\omega^{\varepsilon} \chi+\frac{1}{2} \alpha^{\varepsilon}|\nabla \chi|^{2}+I_{[0,1]}(\chi), \\
& \tilde{\psi}^{\varepsilon}\left(e\left(u^{\varepsilon}\right), \chi\right)=\frac{1}{2}\left[\varepsilon \lambda^{0}(\chi) \operatorname{tr} e\left(u^{\varepsilon}\right)^{2}+2 \varepsilon \mu^{0}(\chi) e\left(u^{\varepsilon}\right)^{2}\right] \quad \text { if } \operatorname{tr}\left(e\left(u^{\varepsilon}\right)\right) \geq 0, \\
& \tilde{\psi}^{\varepsilon}\left(e\left(u^{\varepsilon}\right), \chi\right)=\frac{1}{2}\left[\lambda^{1}(\chi) \operatorname{tr} e\left(u^{\varepsilon}\right)^{2}+2 \varepsilon \mu^{0}(\chi) e\left(u^{\varepsilon}\right)^{2}\right] \quad \text { if } \operatorname{tr}\left(e\left(u^{\varepsilon}\right)\right) \leq 0,
\end{aligned}
$$


where $\omega^{\varepsilon}$ is a positive parameter close to a cohesion parameter as Dupré's energy, ${ }^{28,5}$ $\alpha^{\varepsilon}$ is an interaction coefficient and $\nabla$ is the gradient operator. Moreover all three parameters $\alpha^{\varepsilon}, \eta^{\varepsilon}$ and $\omega^{\varepsilon}$ are supposed inversely proportional to $\varepsilon$. In upcoming section we will take $\alpha^{\varepsilon}=\frac{\alpha}{\varepsilon}, \eta^{\varepsilon}=\frac{\eta}{\varepsilon}$ and $\omega^{\varepsilon}=\frac{\omega}{\varepsilon}$, where $\eta, \alpha$ and $\omega$ are positive parameters. Furthermore in Eq. (6), the indicator function $I$ put physical constraint which ensures that the damage parameter remains in the interval $[0,1]$. Moreover with respect to the given pseudo-Potential (Eq. (5)) and the choice of $\eta^{\varepsilon} \geq 0$ leads to write the following evolution equations in the damage parameter $\chi$ with two regimes. It is observed that the choice of a nonsymmetric behavior in both traction and compression is similar to the choice taken by Mielke et al. ${ }^{29}$ It will be shown that this choice will certify in the limit problem to have unilateral constraint on the interface.

Using classical thermodynamic considerations, the evolution equation is

$$
\begin{cases}\eta^{\varepsilon} \dot{\chi}=\left(\omega^{\varepsilon}-\alpha^{\varepsilon} \Delta \chi-\frac{1}{2}\left[\varepsilon \lambda_{, \chi}^{0} \operatorname{tr} e\left(u^{\varepsilon}\right)^{2}+2 \varepsilon \mu_{, \chi}^{0} e\left(u^{\varepsilon}\right)^{2}\right]\right)_{-} & \text {if } \operatorname{tr}\left(e\left(u^{\varepsilon}\right)\right) \geq 0, \\ \eta^{\varepsilon} \dot{\chi}=\left(\omega^{\varepsilon}-\alpha^{\varepsilon} \Delta \chi-\frac{1}{2}\left[\lambda_{, \chi}^{1} \operatorname{tr} e\left(u^{\varepsilon}\right)^{2}+2 \varepsilon \mu_{, \chi}^{0} e\left(u^{\varepsilon}\right)^{2}\right]\right)_{-} \quad \text { if } \operatorname{tr}\left(e\left(u^{\varepsilon}\right)\right) \leq 0,\end{cases}
$$

where $\Delta$ is Laplacian in space variable and ()$_{-}$is the negative part of a value. Therefore, the equilibrium problem of the damaged composite body is given below,

$$
\left\{\begin{array}{lr}
\sigma_{i j, j}^{\varepsilon}+f_{i}=0 & \text { in } \Omega_{ \pm}^{\varepsilon}, \\
\sigma_{i j}^{\varepsilon} n_{j}=g_{i} & \text { on } S_{g}^{\varepsilon}, \\
\sigma_{i j}^{\varepsilon}=0 & \text { in } B^{\varepsilon}, \\
{\left[\left[\sigma_{i 3}^{\varepsilon}\right]\right]=0} & \text { on } S_{ \pm}^{\varepsilon}, \\
{\left[\left[u_{i}^{\varepsilon}\right]\right]=0} & \text { on } S_{ \pm}^{\varepsilon}, \\
u_{i}^{\varepsilon}=0 & \text { on } S_{u}, \\
\sigma_{i j}^{\varepsilon}=a_{i j h k}^{ \pm} e_{h k}\left(u^{\varepsilon}\right) & \text { in } \Omega_{ \pm}^{\varepsilon}, \\
\sigma^{\varepsilon}=\varepsilon \lambda^{0} \operatorname{tr} e\left(u^{\varepsilon}\right) I_{2}+2 \varepsilon \mu^{0} e\left(u^{\varepsilon}\right) & \text { if } \operatorname{tr} e\left(u^{\varepsilon}\right) \geq 0 \text { in } B^{\varepsilon}, \\
\sigma^{\varepsilon}=\lambda^{1} \operatorname{tr} e\left(u^{\varepsilon}\right) I_{2}+2 \varepsilon \mu^{0} e\left(u^{\varepsilon}\right) & \text { if } \operatorname{tr} e\left(u^{\varepsilon}\right) \leq 0 \text { in } B^{\varepsilon}, \\
\eta^{\varepsilon} \dot{\chi}=\left(\omega^{\varepsilon}+\alpha^{\varepsilon} \Delta \chi-\frac{1}{2}\left[\varepsilon \lambda_{, \chi}^{0} \operatorname{tr} e\left(u^{\varepsilon}\right)^{2}+2 \varepsilon \mu_{, \chi}^{0} e\left(u^{\varepsilon}\right)^{2}\right]\right)_{-} & \text {if } \operatorname{tr} e\left(u^{\varepsilon}\right) \geq 0 \text { in } B^{\varepsilon}, \\
\eta^{\varepsilon} \dot{\chi}=\left(\omega^{\varepsilon}+\alpha^{\varepsilon} \Delta \chi-\frac{1}{2}\left[\lambda_{, \chi}^{1} \operatorname{tr} e\left(u^{\varepsilon}\right)^{2}+2 \varepsilon \mu_{, \chi}^{0} e\left(u^{\varepsilon}\right)^{2}\right]\right)_{-} & \text {if } \operatorname{tr} e\left(u^{\varepsilon}\right) \leq 0 \text { in } B^{\varepsilon}, \\
\chi \in[0,1] & \text { in } B^{\varepsilon}, \\
\dot{\chi} \leq 0 & \text { in } B^{\varepsilon} .
\end{array}\right.
$$

In Eq. (8) the symbol [[ $\varphi]]$ denotes the jump of a function $\varphi$ across $S_{ \pm}^{\varepsilon}$ i.e $\varphi\left(( \pm \varepsilon / 2)^{ \pm}\right)-$ $\varphi\left(( \pm \varepsilon / 2)^{\mp}\right)$, where $\varphi\left(a^{+}\right)=\lim _{x \rightarrow a, x>a} \varphi(x)$ and $\varphi\left(a^{-}\right)=\lim _{x \rightarrow a, x<a} \varphi(x)$

\section{Asymptotic Method}

In this section, we are interested to derive a model of interface for the composite made by the adhesive and the adherents by using asymptotic methods from the 
system of equations Eq. (8). For more detail, one can refer to Refs. 30-37 and references therein. The idea is to replace the interphase by an interface. The following asymptotic expansions in both $u^{\varepsilon}$ and $\sigma^{\varepsilon}$ are defined:

$$
\left\{\begin{array}{l}
u^{\varepsilon}=u^{0}+\varepsilon u^{1}+o(\varepsilon), \\
\sigma^{\varepsilon}=\sigma^{0}+\varepsilon \sigma^{1}+o(\varepsilon) .
\end{array}\right.
$$

\subsection{Rescaling}

Let us bring change of variable in model given in Eq. (8) by using the idea of Ciarlet. ${ }^{30}$ Complete illustration for such change of variable is shown in Fig. 1 (right). At first stage, we will bring change of variable (rescaling) in the adhesive, if $\left(x_{1}, x_{2}, x_{3}\right) \in B^{\varepsilon}$ then $\left(z_{1}, z_{2}, z_{3}\right) \in B$ such that $z_{1}=x_{1}, z_{2}=x_{2}$ and $z_{3}=\frac{x_{3}}{\varepsilon}$. Further change in displacement field and strain can, respectively, be expressed as $u^{\varepsilon}\left(x_{1}, x_{2}\right.$, $\left.x_{3}\right)=\hat{u}^{\varepsilon}\left(z_{1}, z_{2}, z_{3}\right)$ and $\sigma^{\varepsilon}\left(x_{1}, x_{2}, x_{3}\right)=\hat{\sigma} \varepsilon\left(z_{1}, z_{2}, z_{3}\right)$, where $B=\left\{\left(z_{1}, z_{2}, z_{3}\right) \in \Omega\right.$ : $\left.\left|z_{3}\right|<\frac{1}{2}\right\}$ which indicates the domain of the interphase after the change of variable. In the adherents the change of variable (translation) is given as if $\left(x_{1}, x_{2}, x_{3}\right) \in \Omega_{ \pm}^{\varepsilon}$ then $\left(z_{1}, z_{2}, z_{3}\right) \in \Omega_{ \pm}$such that $\left(z_{1}, z_{2}, z_{3}\right)=\left(x_{1}, x_{2}, x_{3} \pm \frac{1}{2}(1-\varepsilon)\right)$, where " + " sign is applied for $\Omega_{+}$and "-" sign is applied for $\Omega_{-}$. Further change in displacement field and strain can be, respectively, expressed as $u^{\varepsilon}\left(x_{1}, x_{2}, x_{3}\right)=\bar{u}^{\varepsilon}\left(z_{1}, z_{2}, z_{3}\right)$ and $\sigma^{\varepsilon}\left(x_{1}, x_{2}, x_{3}\right)=\bar{\sigma}^{\varepsilon}\left(z_{1}, z_{2}, z_{3}\right)$, where $\Omega_{ \pm}=\left\{\left(z_{1}, z_{2}, z_{3}\right) \in \Omega: \pm z_{3}>\frac{1}{2}\right\}$ indicates the domain of adherents after change of variables. As the loadings are acting on the adherents only therefore, they are independent of $\varepsilon$, i.e., the change of variable does not affect them, therefore, $\bar{f}\left(z_{1}, z_{2}, z_{3}\right)=f\left(x_{1}, x_{2}, x_{3}\right)$ and $\bar{g}\left(z_{1}, z_{2}, z_{3}\right)=g\left(x_{1}, x_{2}, x_{3}\right)$. Relations among the derivative of the rescaling are $\frac{\partial}{\partial x_{1}}=\frac{\partial}{\partial z_{1}}, \frac{\partial}{\partial x_{2}}=\frac{\partial}{\partial z_{2}}$ and $\frac{\partial}{\partial x_{3}}=\frac{1}{\varepsilon} \frac{\partial}{\partial z_{3}}$. Moreover, the system of Eq. (8) rescaled can be written as

$$
\left\{\begin{array}{lc}
\bar{\sigma}_{i j, j}^{\varepsilon}+\bar{f}_{i}=0 & \text { in } \Omega_{ \pm}, \\
\bar{\sigma}_{i j}^{\varepsilon} n_{j}=g_{i} & \text { on } \bar{S}_{g}, \\
\hat{\sigma}_{i j}^{\varepsilon}=0 & \text { in } B, \\
\bar{\sigma}_{i 3}^{\varepsilon}=\hat{\sigma}_{i 3}^{\varepsilon} & \text { on } S_{ \pm}, \\
\bar{u}_{i}^{\varepsilon}=\hat{u}_{i}^{\varepsilon} & \text { on } S_{ \pm}, \\
\bar{u}_{i}^{\varepsilon}=0 & \text { on } \bar{S}_{u}, \\
\bar{\sigma}_{i j}^{\varepsilon}=a_{i j h k}^{ \pm} \bar{e}_{h k}\left(\bar{u}^{\varepsilon}\right) & \text { in } \Omega_{ \pm}, \\
\hat{\sigma}_{\varepsilon}^{\varepsilon}=\varepsilon \lambda^{0}(\chi) \operatorname{tr} \hat{e}\left(\hat{u}^{\varepsilon}\right) I_{2}+2 \varepsilon \mu^{0}(\chi) \hat{e}\left(\hat{u}^{\varepsilon}\right) & \text { if } \operatorname{tr} \hat{e}\left(\hat{u}^{\varepsilon}\right) \geq 0 \text { in } B, \\
\hat{\sigma}_{\varepsilon}=\lambda^{1}(\chi) \operatorname{tr} \hat{e}\left(\hat{u}^{\varepsilon}\right) I_{2}+2 \varepsilon \mu^{0}(\chi) \hat{e}\left(\hat{u}^{\varepsilon}\right) & \text { if } \operatorname{tr} \hat{e}\left(\hat{u}^{\varepsilon}\right) \leq 0 \text { in } B, \\
\hat{\eta}^{\varepsilon} \dot{\hat{\chi}}=\left(\hat{\omega} \varepsilon-\hat{\alpha} \varepsilon \Delta \chi-\frac{1}{2}\left[\varepsilon \lambda_{, \chi}^{0} \operatorname{tr} \hat{e}\left(\hat{u}^{\varepsilon}\right)^{2}+2 \varepsilon \mu_{, \chi}^{0} \hat{e}\left(\hat{u}^{\varepsilon}\right)^{2}\right]\right)_{-} & \text {if } \operatorname{tr} \hat{e}\left(\hat{u}^{\varepsilon}\right) \geq 0 \text { in } B, \\
\hat{\eta}^{\varepsilon} \dot{\hat{\chi}}=\left(\hat{\omega} \varepsilon-\hat{\alpha} \varepsilon \Delta \chi-\frac{1}{2}\left[\lambda_{, \chi}^{1} \operatorname{tr} \hat{e}\left(\hat{u}^{\varepsilon}\right)^{2}+2 \varepsilon \mu_{, \chi}^{0} \hat{e}\left(\hat{u}^{\varepsilon}\right)^{2}\right]\right)_{-} & \text {if } \operatorname{tr} \hat{e}\left(\hat{u}^{\varepsilon}\right) \leq 0 \text { in } B, \\
\hat{\chi} \in[0,1] & \text { in } B, \\
\dot{\hat{\chi}} \leq 0 & \text { in } B,
\end{array}\right.
$$


where $\hat{\text {. and }}{ }^{-}$denotes, respectively the change of variable in adherents and adhesive and $S_{ \pm}=\left\{\left(z_{1}, z_{2}, z_{3}\right) \in \Omega: z_{3}= \pm \frac{1}{2}\right\}$ indicates the interface between $B$ and $\Omega_{ \pm}$.

Asymptotic expansion of displacement field and stress field, after rescaling can be written as

$$
\left\{\begin{array}{l}
\hat{u}^{\varepsilon}=\hat{u}^{0}+\varepsilon \hat{u}^{1}+o(\varepsilon), \\
\hat{\sigma} \varepsilon=\hat{\sigma}^{0}+\varepsilon \hat{\sigma}^{1}+o(\varepsilon), \\
\bar{u}^{\varepsilon}=\bar{u}^{0}+\varepsilon \bar{u}^{1}+o(\varepsilon), \\
\bar{\sigma}^{\varepsilon}=\bar{\sigma}^{0}+\varepsilon \bar{\sigma}^{1}+o(\varepsilon) .
\end{array}\right.
$$

\subsection{Asymptotic expansion for equilibrium model for adherents}

In a similar way as done by Bonetti et al., ${ }^{16}$ we substitute asymptotic expansion Eq. (11) in first, second, sixth and seventh equation of Eq. (10) and obtain at firstorder (power 0) the problem given below,

$$
\begin{cases}\bar{\sigma}_{i j, j}^{0}+\bar{f}_{i}=0 & \text { in } \Omega_{ \pm}, \\ \bar{\sigma}_{i j}^{0} n_{j}=g_{i} & \text { on } \bar{S}_{g}, \\ \bar{u}_{i}^{0}=0 & \text { on } \bar{S}_{u}, \\ \bar{\sigma}_{i j}^{0}=a_{i j h k}^{ \pm} \bar{e}_{h k}\left(\bar{u}^{0}\right) & \text { in } \Omega_{ \pm} .\end{cases}
$$

At this level, we have to find conditions on the internal interface $S$ to close the problem.

\subsection{Asymptotic expansion for equilibrium equation for adhesive}

In the same way, substituting asymptotic expansions Eq. (11) in the third equation of Eq. (10), we can get $o\left(\varepsilon^{-1}\right)$-problem,

$$
\hat{\sigma}_{i 3,3}^{0}=0 .
$$

It shows that the stress vector $\hat{\sigma}_{i 3}^{0}$ does not depend on $z_{3}$ then, Eq. (13) can be written as

$$
\left[\hat{\sigma}_{i 3}^{0}\right]=0,
$$

where symbol [.] is defined as, $[f]=f\left(z_{1}, z_{2}, \frac{1}{2}\right)-f\left(z_{1}, z_{2},-\frac{1}{2}\right)$, i.e., it indicates the jumps between $z_{3}=+\frac{1}{2}$ and $z_{3}=-\frac{1}{2}$. Strain field in the adhesive with respect to the choice of Rizzoni et al. ${ }^{37}$ is used as

$$
\hat{e}\left(\hat{u}^{\varepsilon}\right)=\varepsilon^{-1} \hat{e}^{-1}+\hat{e}^{0}+\varepsilon \hat{e}^{1}+o(\varepsilon),
$$

where

$$
\left\{\begin{array}{l}
\hat{e}_{33}^{-1}=\hat{u}_{3,3}^{0}, \\
\hat{e}_{b 3}^{-1}=\frac{1}{2} \hat{u}_{b, 3}^{0} \quad \text { for } b=1,2 .
\end{array}\right.
$$


The trace of the strain in the adhesive is remarked as,

$$
\operatorname{tr} \hat{e}\left(\hat{u}^{\varepsilon}\right)=\varepsilon^{-1} \hat{u}_{3,3}^{0}+\varepsilon^{0}\left(\hat{u}_{1,1}^{0}+\hat{u}_{2,2}^{0}+\hat{u}_{3,3}^{1}\right)+o(\varepsilon) .
$$

Thus,

$$
\hat{u}_{3,3}^{0} \leq 0
$$

and

$$
\hat{u}_{1,1}^{0}+\hat{u}_{2,2}^{0}+\hat{u}_{3,3}^{1} \leq 0 .
$$

Substituting Eq. (11) in eighth equation of Eq. (10), and collecting (power-0) gives $o(1)$-problem,

$$
\hat{\sigma}_{i 3}^{0}=\lambda^{0}(\chi) \hat{u}_{3,3}^{0} \delta_{i, 3}+2 \mu^{0}(\chi) \hat{e}_{i 3}^{-1} \quad \text { for } i=1,2,3
$$

where $\delta_{i, 3}$ is the Kronecker symbol. Moreover integrating Eq. (20) along $z_{3}$, we have

$$
\left\{\begin{array}{l}
\hat{\sigma}_{b 3}^{0}=\left\langle\mu^{0}(\chi)\right\rangle_{*}\left[\hat{u}_{i}^{0}\right] \quad \text { for } b=1,2, \\
\hat{\sigma}_{33}^{0}=\left\langle\lambda^{0}(\chi)+2 \mu^{0}(\chi)\right\rangle_{*}\left[\hat{u}_{3}^{0}\right],
\end{array}\right.
$$

where $\left\langle\mu^{0}(\chi)\right\rangle_{*}=\left[\int_{\frac{-1}{2}}^{\frac{1}{2}} \frac{d z_{3}}{\mu^{0}(\chi)}\right]^{-1}$ and $\left\langle\lambda^{0}(\chi)+2 \mu^{0}(\chi)\right\rangle_{*}=\left[\int_{\frac{-1}{2}}^{\frac{1}{2}} \frac{d z_{3}}{\lambda^{0}(\chi)+2 \mu^{0}(\chi)}\right]^{-1}$.

For example, $\hat{\sigma}_{13}^{0}=\mu^{0}(\chi) \hat{u}_{i, 3}^{0}$. Then $\hat{u}_{i, 3}^{0}=\frac{\hat{\sigma}_{13}^{0}}{\mu^{0}(\chi)}$ and by integrating along $z_{3}$, it is obtained Eq. (21).

Equation (21) is the classical equation for soft interface for traction, i.e., when $\left[\hat{u}_{3}^{0}\right] \geq 0$.

For compression, substituting Eq. (11) in the ninth equation of Eq. (10) (order $\left.o\left(\varepsilon^{-1}\right)\right)$, it is obtained,

$$
0=\lambda^{1}(\chi) \hat{u}_{3,3}^{0}, \quad \text { if } \hat{u}_{3,3}^{0} \leq 0
$$

In other words, due to the positivity of $\lambda,{ }^{1}$

$$
\hat{u}_{3,3}=0, \quad \text { if } \hat{u}_{3,3} \leq 0 .
$$

If $\hat{u}_{3,3}$ being independent of $z_{3}$, then the jump can be written as

$$
\left[\hat{u}_{3}\right]=0, \quad \text { if }\left[\hat{u}_{3}\right] \leq 0
$$

and

$$
\hat{\sigma}_{33}^{0}=\lambda^{1}(\chi)\left(\hat{u}_{1,1}^{0}+\hat{u}_{2,2}^{0}+\hat{u}_{3,3}^{1}\right) \leq 0
$$

or

$$
\hat{\sigma}_{33}^{0} \leq 0
$$


Equation (24) shows that in compression there is no jump on the interface in the third direction and Eq. (25) indicates that in the compression case the third component of the stress vector is negative along the interface (no adhesion).

In this paper we denote $\Delta_{2} \chi=\chi_{, 11}+\chi_{, 22}$, the Laplacian in the plane $\left(z_{1}, z_{2}\right)$. Substituting Eq. (11) in the ninth equation of the Eq. (10), and collecting the same powers (power-3) and (power-1), respectively gives

$$
\chi_{, 33}=0
$$

and

$$
\hat{\eta} \dot{\chi}=\left(\hat{\omega}-\hat{\alpha} \Delta_{2} \chi-\frac{1}{2}\left(\lambda_{, \chi}^{0}(\chi)+2 \mu_{, \chi}^{0}(\chi)\right)\left(\hat{u}_{3,3}^{0}\right)^{2}-\frac{1}{2} \mu_{, \chi}^{0}(\chi)\left\{\left(\hat{u}_{1,3}^{0}\right)^{2}+\left(\hat{u}_{2,3}^{0}\right)^{2}\right\}\right) .
$$

Similarly (for compression) substituting Eq. (11) in tenth equation of the Eq. (10) and collecting the same powers (power-3), (power-2) and (power-1), receptively gives following results:

$$
\begin{gathered}
\chi_{, 33}=0 \\
0=-\frac{1}{2} \lambda_{, \chi}^{1}(\chi)\left(\hat{u}_{3,3}^{0}\right)^{2}
\end{gathered}
$$

or

$$
\hat{u}_{3,3}^{0}=0
$$

and

$$
\hat{\eta} \dot{\chi}=\hat{\omega}-\hat{\alpha}^{-1} \Delta_{2} \chi-\mu_{, \chi}^{0}(\chi)\left\{\frac{1}{2}\left(\hat{u}_{1,3}^{0}\right)^{2}+\frac{1}{2}\left(\hat{u}_{2,3}^{0}\right)^{2}+\left(\hat{u}_{3,3}^{0}\right)^{2}\right\} .
$$

Substituting Eq. (31) in Eq. (32), we get

$$
\hat{\eta} \dot{\chi}=\left(\hat{\omega}-\hat{\alpha} \Delta_{2} \chi-\frac{1}{2} \mu_{, \chi}^{0}(\chi)\left\{\left(\hat{u}_{1,3}^{0}\right)^{2}+\left(\hat{u}_{2,3}^{0}\right)^{2}\right\}\right)_{-} .
$$

3.3.1. Case 1: $\chi$ does not depend on $z_{3}$ and therefore, $\chi_{, 3}=0$.

Equations (27) and (29) indicates that along $z_{3}$ Laplacian component is equal to zero in both traction and compression. Further in this case we assumed $\chi_{, 3}=0$, integrating Eq. (27) or Eq. (29) two times along $z_{3}$ gives,

$$
\chi=c \text {. }
$$

Further as we took negative evolution of damage, i.e., $\chi$ never increases and in this case as $\chi$ does not depend on $z_{3}$, therefore, we can write, $\left\langle\mu^{0}(\chi)\right\rangle_{*}=\mu^{0}(\chi)$ and $\left\langle\lambda^{0}(\chi)+2 \mu^{0}(\chi)\right\rangle_{*}=\lambda^{0}(\chi)+2 \mu^{0}(\chi)$, then integrating Eqs. (28) and (33) along $z_{3}$ by considering Eq. (21) in two steps we obtain the equation of motion in traction and 
compression, respectively as follows:

$$
\begin{gathered}
\hat{\eta} \dot{\chi}=\left(\hat{\omega}-\hat{\alpha} \Delta_{2} \chi-\frac{1}{2}\left\{\lambda_{, \chi}^{0}(\chi)+2 \mu_{, \chi}^{0}(\chi)\right\}\left[\hat{u}_{3}^{0}\right] \cdot\left[\hat{u}_{3}^{0}\right]\right. \\
\left.-\frac{1}{2} \mu_{, \chi}^{0}(\chi)\left\{\left[\hat{u}_{1}^{0}\right] \cdot\left[\hat{u}_{1}^{0}\right]+\left[\hat{u}_{2}^{0}\right] \cdot\left[\hat{u}_{2}^{0}\right]\right\}\right)_{-} \text {if }\left[\hat{u}_{3}\right] \geq 0 . \\
\hat{\eta} \dot{\chi}=\left(\hat{\omega}-\hat{\alpha} \Delta_{2} \chi-\frac{1}{2} \mu_{, \chi}^{0}(\chi)\left\{\left[\hat{u}_{1}^{0}\right] \cdot\left[\hat{u}_{1}^{0}\right]+\left[\hat{u}_{2}^{0}\right] \cdot\left[\hat{u}_{2}^{0}\right]\right\}\right)_{-} \quad \text { if }\left[\hat{u}_{3}\right] \leq 0 .
\end{gathered}
$$

\subsubsection{Case 2: $\chi$ depends on $z_{3}$ and $\chi_{, 3} \neq 0$}

As in this case we suppose that $\chi_{, 3} \neq 0$ then by integrating Eq. (27) or Eq. (29) two times along $z_{3}$ gives

$$
\chi=c_{1} z_{3}+c_{2},
$$

where $c_{1}, c_{2}$ are constants of integration. Eq. (37) can also be written as

$$
\chi=[\chi] z_{3}+\langle\langle\chi\rangle\rangle
$$

where $c_{1}=\chi\left(z_{1}, z_{2}, \frac{1}{2}\right)-\chi\left(z_{1}, z_{2}, \frac{-1}{2}\right)=[\chi]$ and $c_{2}=\frac{\chi\left(z_{1}, z_{2}, \frac{1}{2}\right)+\chi\left(z_{1}, z_{2}, \frac{-1}{2}\right)}{2}=\langle\langle\chi\rangle\rangle$. We denote $\langle\rangle=.\int_{\frac{-1}{2}}^{\frac{1}{2}}(.) d z_{3}$. Integrating Eq. (38) for $\frac{-1}{2}$ to $\frac{1}{2}$ along $z_{3}$ gives,

$$
\langle\chi\rangle=\int_{\frac{-1}{2}}^{\frac{1}{2}} \chi d z_{3}=\langle\langle\chi\rangle\rangle .
$$

By substituting Eq. (20) in Eq. (28) and in Eq. (32), equilibrium equation in traction and compression, respectively reads as

$$
\begin{aligned}
\hat{\eta} \dot{\chi}= & \hat{\omega}-\hat{\alpha} \Delta_{2} \chi-\frac{1}{2}\left\{\frac{\lambda_{, \chi}^{0}(\chi)+2 \mu_{, \chi}^{0}(\chi)}{\lambda^{0}(\chi)+2 \mu^{0}(\chi)}\right\} \hat{\sigma}_{33}^{0} \hat{u}_{3,3}^{0}-\frac{1}{2}\left\{\frac{\mu_{, \chi}^{0}(\chi)}{\mu^{0}(\chi)}\right\} \hat{\sigma}_{13}^{0} \hat{u}_{1,3}^{0} \\
& -\frac{1}{2}\left\{\frac{\mu_{, \chi}^{0}(\chi)}{\mu^{0}(\chi)}\right\} \hat{\sigma}_{23}^{0} \hat{u}_{2,3}^{0} . \\
& \hat{\eta} \dot{\chi}=\hat{\omega}-\hat{\alpha} \Delta_{2} \chi-\frac{1}{2}\left\{\frac{\mu_{, \chi}^{0}(\chi)}{\mu^{0}(\chi)}\right\} \hat{\sigma}_{13}^{0} \hat{u}_{1,3}^{0}-\frac{1}{2}\left\{\frac{\mu_{, \chi}^{0} \chi}{\mu^{0}(\chi)}\right\} \hat{\sigma}_{23}^{0} \hat{u}_{2,3}^{0} .
\end{aligned}
$$

Again substituting Eq. (20) in Eqs. (40) and (41) we can get following equations:

$$
\begin{aligned}
\hat{\eta} \dot{\chi}= & \hat{\omega}-\hat{\alpha} \Delta_{2} \chi-\frac{1}{2}\left\{\frac{\lambda_{, \chi}^{0}(\chi)+2 \mu_{, \chi}^{0}(\chi)}{\left(\lambda^{0}(\chi)+2 \mu^{0}(\chi)\right)^{2}}\right\} \hat{\sigma}_{33}^{0} \hat{\sigma}_{33}^{0}-\frac{1}{2}\left\{\frac{\mu_{, \chi}^{0}(\chi)}{\left(\mu^{0}(\chi)\right)^{2}}\right\} \hat{\sigma}_{13}^{0} \hat{\sigma}_{13}^{0} \\
& -\frac{1}{2}\left\{\frac{\mu_{, \chi}^{0}(\chi)}{\left(\mu^{0}(\chi)\right)^{2}}\right\} \hat{\sigma}_{23}^{0} \hat{\sigma}_{23}^{0} . \\
& \hat{\eta} \dot{\chi}=\hat{\omega}-\hat{\alpha} \Delta_{2} \chi-\frac{1}{2}\left\{\frac{\mu_{, \chi}^{0}(\chi)}{\left(\mu^{0}(\chi)\right)^{2}}\right\} \hat{\sigma}_{23}^{0} \hat{\sigma}_{33}^{0}-\frac{1}{2}\left\{\frac{\mu_{, \chi}^{0}(\chi)}{\left(\mu^{0}(\chi)\right)^{2}}\right\} \hat{\sigma}_{23}^{0} \hat{\sigma}_{23}^{0} .
\end{aligned}
$$


Integrating Eq. (42), which is the equation of motion in traction, along $z_{3}$, and using Eq. (21) gives,

$$
\begin{aligned}
\hat{\eta}\langle\dot{\chi}\rangle= & \hat{\omega}-\hat{\alpha} \Delta_{2}\langle\chi\rangle-\frac{1}{2}\left\langle\frac{\lambda_{, \chi}^{0}(\chi)+2 \mu_{, \chi}^{0}(\chi)}{\left(\lambda^{0}(\chi)+2 \mu^{0}(\chi)\right)^{2}}\right\rangle\left\langle\lambda^{0}(\chi)+2 \mu^{0}(\chi)\right\rangle_{*}^{2}\left[\hat{u}_{3}^{0}\right] \cdot\left[\hat{u}_{3}^{0}\right] \\
& -\frac{1}{2}\left\{\left\langle\frac{\mu_{, \chi}^{0}(\chi)}{\left(\mu^{0}(\chi)\right)^{2}}\right\rangle\left\langle\mu^{0}(\chi)\right\rangle_{*}^{2}\left[\hat{u}_{1}^{0}\right] \cdot\left[\hat{u}_{1}^{0}\right]+\left\langle\frac{\mu_{, \chi}^{0}(\chi)}{\left(\mu^{0}(\chi)\right)^{2}}\right\rangle\left\langle\mu^{0}(\chi)\right\rangle_{*}^{2}\left[\hat{u}_{2}^{0}\right] \cdot\left[\hat{u}_{2}^{0}\right]\right\} .
\end{aligned}
$$

In order to simplify last expression, we introduce two results.

Lemma 1. Let $F \in C^{1}([0,1] ; \mathbb{R})$, let $G \in C^{0}([-1 / 2,1 / 2] ;[0,1])$ such that $G\left(z_{3}\right)=$ $a z_{3}+b$ then $\left\langle F_{, G}(G)\right\rangle=\left\langle F_{, b}(G)\right\rangle$ and $\langle F(G)\rangle_{, b}=\left\langle F_{, G}(G)\right\rangle$.

\section{Proof.}

$$
\begin{aligned}
\left\langle F_{, G}(G)\right\rangle & =\left\langle F^{\prime}\left(a z_{3}+b\right)\right\rangle=\frac{1}{a}(F(a / 2+b)-F(-a / 2+b)), \\
\left\langle F_{, b}(G)\right\rangle & =\left\langle F^{\prime}\left(a z_{3}+b\right)\right\rangle=\frac{1}{a}(F(a / 2+b)-F(-a / 2+b)), \\
\langle F(G)\rangle_{, b} & =\left(\frac{1}{a}\left(\left(\int F\right)(a / 2+b)-\left(\int F\right)(-a / 2+b)\right)\right)_{, b} \\
& =\frac{1}{a}(F(a / 2+b)-F(-a / 2+b)) .
\end{aligned}
$$

Lemma 2. Let $F \in C^{1}([0,1] ; \mathbb{R})$, let $G \in C^{0}([-1 / 2,1 / 2] ;[0,1])$ such that $G\left(z_{3}\right)=$ $a z_{3}+b$ then $\langle F(G)\rangle_{*, b}=\langle F(G)\rangle_{*}^{2}\left\langle\frac{F_{, G}(G)}{(F(G))^{2}}\right\rangle$

Proof. Using Lemma 1

$$
\langle F(G)\rangle_{*, b}=\langle F(G)\rangle_{*}^{2}\left\langle\frac{F_{, b}(G)}{(F(G))^{2}}\right\rangle=\langle F(G)\rangle_{*}^{2}\left\langle\frac{F_{, G}(G)}{(F(G))^{2}}\right\rangle .
$$

Using the last lemma, it is obtained for example

$$
\left\langle\mu^{0}(\chi)\right\rangle_{*,\langle\chi\rangle}=\left\langle\mu^{0}(\chi)\right\rangle_{*}^{2}\left\langle\frac{\mu_{, \chi}^{0}(\chi)}{\left(\mu^{0}(\chi)\right)^{2}}\right\rangle .
$$

Similarly, integrating Eq. (43) which is equation of motion in compression along $z_{3}$, and making the use of Eq. (21) result gives

$$
\begin{aligned}
\hat{\eta}\langle\dot{\chi}\rangle= & \hat{\omega}-\hat{\alpha} \Delta_{2}\langle\chi\rangle-\frac{1}{2}\left\langle\frac{\mu_{\chi}^{0}(\chi)}{\left(\mu^{0}(\chi)\right)^{2}}\right\rangle\left\langle\mu^{0}(\chi)\right\rangle_{*}^{2}\left[\hat{u}_{1}^{0}\right] \cdot\left[\hat{u}_{1}^{0}\right] \\
& -\frac{1}{2}\left\langle\frac{\mu_{\chi}^{0}(\chi)}{\left(\mu^{0}(\chi)\right)^{2}}\right\rangle\left\langle\mu^{0}(\chi)\right\rangle_{*}^{2}\left[\hat{u}_{2}^{0}\right] \cdot\left[\hat{u}_{2}^{0}\right] .
\end{aligned}
$$


Then by applying above lemmas, Eq. (44) and Eq. (47) can be re-read, receptively,

$$
\begin{aligned}
\hat{\eta}\langle\dot{\chi}\rangle= & \left(\hat{\omega}-\hat{\alpha} \Delta_{2}\langle\chi\rangle-\frac{1}{2}\left\langle\lambda^{0}(\chi)+2 \mu^{0}(\chi)\right\rangle_{*,\langle\chi\rangle}\left[\hat{u}_{3}^{0}\right] \cdot\left[\hat{u}_{3}^{0}\right]\right. \\
& \left.-\frac{1}{2}\left\{\left\langle\mu^{0}(\chi)\right\rangle_{*,\langle\chi\rangle}\left[\hat{u}_{1}^{0}\right] \cdot\left[\hat{u}_{1}^{0}\right]+\left\langle\mu^{0}(\chi)\right\rangle_{*,\langle\chi\rangle}\left[\hat{u}_{2}^{0}\right] \cdot\left[\hat{u}_{2}^{0}\right]\right\}\right)_{-} \quad \text { if }\left[\hat{u}_{3}\right] \geq 0
\end{aligned}
$$

and

$$
\begin{aligned}
\hat{\eta}\langle\dot{\chi}\rangle= & \left(\hat{\omega}-\hat{\alpha} \Delta_{2}\langle\chi\rangle-\frac{1}{2}\left\{\left\langle\mu^{0}(\chi)\right\rangle_{*,\langle\chi\rangle}\left[\hat{u}_{1}^{0}\right] \cdot\left[\hat{u}_{1}^{0}\right]\right.\right. \\
& \left.\left.+\left\langle\mu^{0}(\chi)\right\rangle_{*,\langle\chi\rangle}\left[\hat{u}_{2}^{0}\right] \cdot\left[\hat{u}_{2}^{0}\right]\right\}\right)_{-} \text {if }\left[\hat{u}_{3}\right] \leq 0 .
\end{aligned}
$$

\subsection{Matching}

The assumption of perfect bonding between adherents and adhesive allows us to use the matching relationship to their stress and displacement fields on the interface $S_{ \pm}$, and such matching can be obtain by substitution of Eqs. (13) in fourth and fifth equation of Eq. (10), we obtain the following relationships:

$$
\hat{\sigma}_{i 3}^{0}\left(z_{1}, z_{2}, \pm \frac{1}{2}\right)=\bar{\sigma}_{i 3}^{0}\left(z_{1}, z_{2}, \pm \frac{1}{2}\right)=\sigma_{i 3}^{0}\left(z_{1}, z_{2}, \pm \frac{\varepsilon}{2}\right) \approx \sigma_{i 3}^{0}\left(x_{1}, x_{2}, 0\right)
$$

and

$$
\hat{u}_{i 3}^{0}\left(z_{1}, z_{2}, \pm \frac{1}{2}\right)=\bar{u}_{i 3}^{0}\left(z_{1}, z_{2}, \pm \frac{1}{2}\right)=u_{i 3}^{0}\left(z_{1}, z_{2}, \pm \frac{\varepsilon}{2}\right) \approx u_{i 3}^{0}\left(x_{1}, x_{2}, 0^{ \pm}\right) .
$$

Finally, the following result is obtained:

$$
\left\{\begin{array}{lc}
\sigma_{i j, j}^{0}+f_{i}=0 & \text { in } \Omega_{ \pm}, \\
\sigma_{i j}^{0} n_{j}=g_{i} & \text { on } S_{g}, \\
u_{i}^{0}=0 & \text { on } S_{u}, \\
\sigma_{i j}^{0}=a_{i j h k}^{ \pm} e_{h k}\left(u^{0}\right) & \text { in } \Omega_{ \pm}, \\
\sigma^{0} e_{3}=K^{*}(\langle\chi\rangle)\left[u^{0}\right]_{+}+p^{0} e_{3} & \text { on } S, \\
p^{0}\left[u_{3}^{0}\right]=0,\left[u_{3}^{0}\right] \geq 0, p^{0} \leq 0 & \text { on } S, \\
\eta\langle\dot{\chi}\rangle=\left(\omega-\alpha \Delta_{2}\langle\chi\rangle-\frac{1}{2} K_{,\langle\chi\rangle}^{*}\left[u^{0}\right]_{+} \cdot\left[u^{0}\right]_{+}\right)_{-} & \text {on } S,
\end{array}\right.
$$

where

$$
K^{*}=\left(\begin{array}{ccc}
\left\langle\mu^{0}\right\rangle_{*} & 0 & 0 \\
0 & \left\langle\mu^{0}\right\rangle_{*} & 0 \\
0 & 0 & \left\langle\lambda^{0}+2 \mu^{0}\right\rangle_{*}
\end{array}\right)
$$


and

$$
\begin{cases}{\left[u^{0}\right]_{+}=\left(\left[u_{1}^{0}\right],\left[u_{2}^{0}\right],\left[u_{3}^{0}\right]\right)^{T}} & \text { if }\left[u_{3}^{0}\right] \geq 0 \\ {\left[u^{0}\right]_{+}=\left(\left[u_{1}^{0}\right],\left[u_{2}^{0}\right], 0\right)^{T}} & \text { if }\left[u_{3}^{0}\right] \leq 0 .\end{cases}
$$

It is obtained a model of imperfect interface with damage evolution and unilateral constraints (Signorini's conditions). Note that "case 1" is a particular case of "case 2 ". It should also be noted that the proposed formulation is in the context of quasistatic problems. In the following, the variable " $\mathrm{t}$ " represents the pseudo-time.

\section{Numerical Example}

Consider an uniform one dimensional rod with negligible body force and with unit cross sectional area. The rod is bonded with a glue on a left support and a traction $f$ is applied on the right of the rod along its cross section area as shown in Fig. 2.

The displacement field is governed by

$$
u(x)=\frac{f(t)}{E} x+u_{0},
$$

where $E$ is the Young's modulus and $u_{0}$ is given by the following equation of interface law:

$$
\sigma=f(t)=E^{*}(\chi) u_{0},
$$

where $E^{*}(\chi)$ is the elastic modulus of the adhesive which is function of $\chi$. We are going to analyse the damage behavior when time increases by assigning different values to the elastic modulus. We recall that it is considered that $\chi=1$ for safe material and $\chi=0$ when the bond is completely damaged and intermediate state when $\chi \in[0,1]$. We choose some examples of $E^{*}(\chi)$ such that $\left\{\begin{array}{l}E^{*}(1)=1 \\ E^{*}(0)=0\end{array}\right.$. Let $E^{*}(\chi)=$ $E_{0} F(\chi)$ and the damage rate which is given by

$$
\eta \dot{\chi}=\left(\omega-\frac{1}{2} E_{, \chi}^{*}(\chi) u_{0}^{2}\right)_{-} .
$$

It is assumed that when $\omega \geq \frac{1}{2} E_{, \chi}^{*}(\chi) u_{0}^{2}$ then $\left\{\begin{array}{c}\chi=1 \\ \dot{\chi}=0\end{array}\right.$. Let us study the behavior of the damage when $\omega \leq \frac{1}{2} E_{, \chi}^{*}(\chi) u_{0}^{2}$. We use Eqs. (54) and (55) and suppose $f(t)=t$

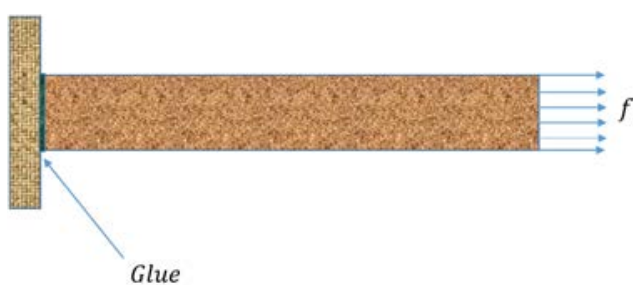

Fig. 2. A simple rod with damaged interface on its left end. 
Table 1. Value of the critical time for various damage functions.

\begin{tabular}{lccc}
\hline$F(\chi)$ & $2^{\chi}-1$ & $\chi^{2}$ & $\chi^{3}$ \\
\hline Critical time $t_{0}$ & 0.17 & 0.1 & 0.082 \\
\hline
\end{tabular}

Note: Different function $F(\chi)$ gives different critical time

which indicates an increasing traction when time increases. For $E^{*}(\chi)=E_{0} F(\chi)$ critical time is obtained $t_{0}=\sqrt{\frac{2 \omega E_{0}}{F^{\prime}(\chi)}} F(\chi)$, i.e., if $t \leq t_{0}$ then bond is undamaged and if $t \geq t_{0}$ then bond starts damaging. Further, suppose that $F(\chi)=2^{\chi}-1, \chi^{2}, \chi^{3}$, $\eta=1, E_{0}=1$, initial condition $\chi\left(t_{0}\right)=\chi(0)=1$ and $\omega=0.01$ then critical time for these functions is given in Table 1.

Further using Explicit Euler scheme at $\Delta t=0.0005$ through Matlab, complete behavior of the interface is shown in Fig. 3 for $t \in[0,0.8]$.

All three curves in Fig. 3 show that as force increases in time, damage in the bond increases and tends to zero (totally damaged interface). The damage evolution depends strongly on the Young modulus dependence in the damage function.

For $F(\chi)=\chi^{2}$ let us see the relationship between the stress and the jump in the displacements for various values of viscosity which is illustrated in Fig. 4 in time $t \in[0,0.3]$. We assume that before the critical time (0.1), i.e., when the stress and jump of displacement are linear then slope in equal to 1 (one). Moreover after the critical time we assume $\omega=0.01001$. Note that when $\eta=0$ then $\chi=\frac{\omega}{\left[u_{0}\right]^{2}}$. When $\eta$ is

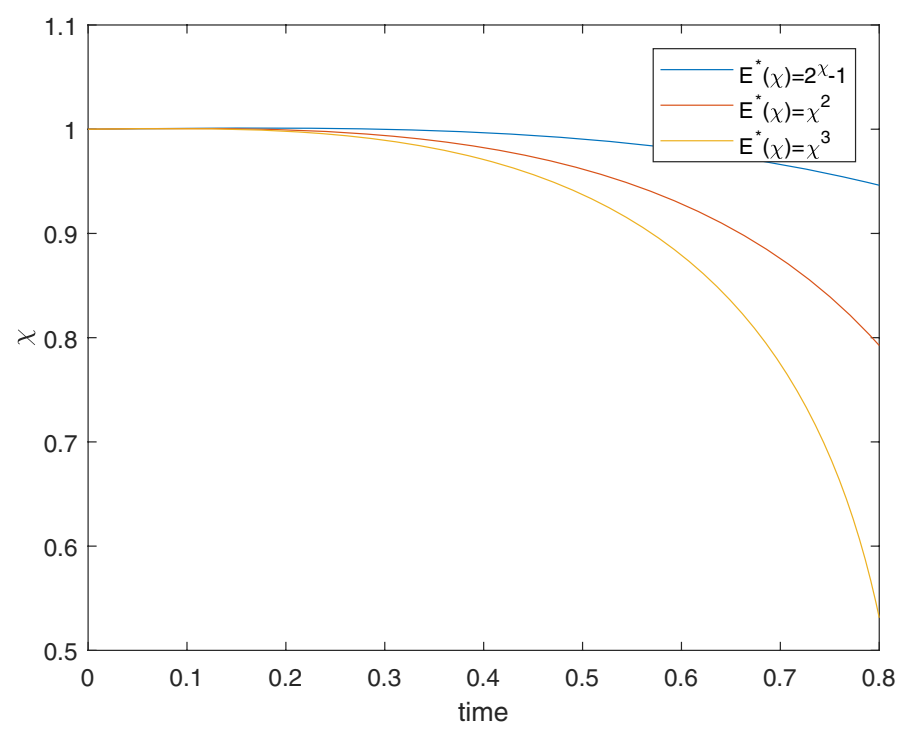

Fig. 3. Evolution of damage. 


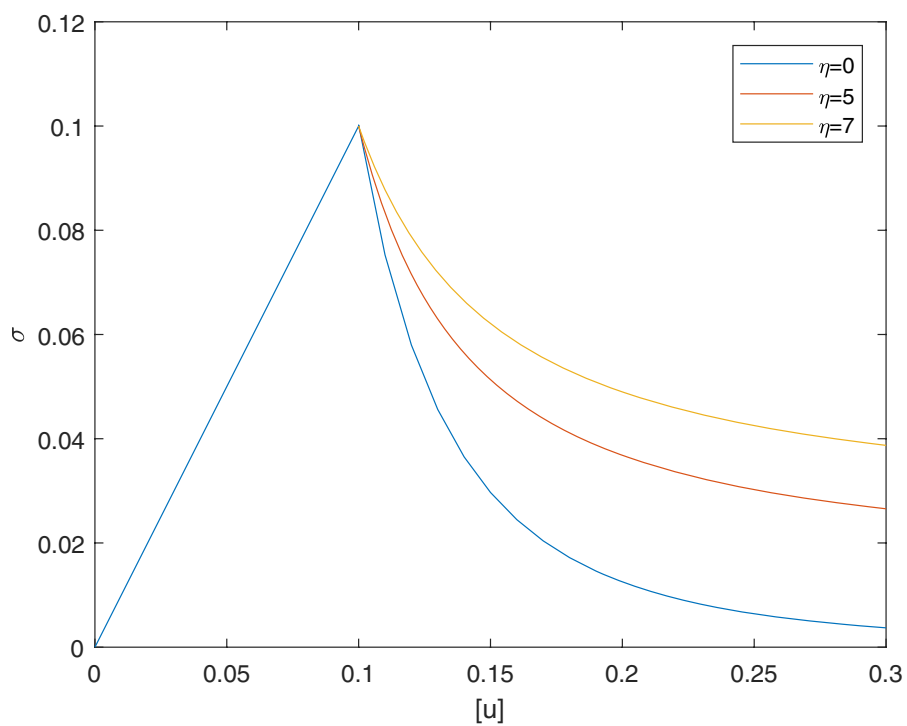

Fig. 4. Behaverial relationship between stress and jump of displacement for different values of viscosity.

positive we used Euler Explicit scheme to solve the differential equation. It is observed a large dependence of the displacement/stress relationship on this coefficient.

\section{Conclusion}

By a technique of homogenization and using thermodynamics considerations at the microscopic level we proposed nonlocal damaged mechanical model of composite structure made by isotopic material. We derived imperfect interface model from the damage mechanical model proposed initially by using the matching asymptotic expansions method and considering perfect bonding conditions between the adherents and the adhesive. Further, we discussed two cases according to dependency of damage evolution along the interphase's thickness. A model of imperfect interface with damage evolution and non penetration condition is obtained. We gave a numerical example to study qualitatively the evolutions of the model.

In the future, we intend to develop the model and implement it considering uncertainties and nonlinearities in the materials (adhesive and adherents).

\section{References}

1. Y. Benveniste and T. Miloh, Imperfect soft and stiff interfaces in two-dimensional elasticity, Mech. Mater. 33 (2001) 309-323.

2. P. G. Bornert, T. Bretheau and P. Gilormini, Homogeńeísation en mećanique des materiaux, Tome 1: Materiaux aleátoires élastiques et milieux peŕiodiques, Hermes Sciences, Paris (2001).

3. N. Challamel and U. A. Girhammar, Boundary-layer effect in composite beams with interlayer slip, J. Aerospace Eng. 24 (2011) 199-209. 
4. M. Frémond, Adhérence des solides, J. de Mécanique Théorique et Appliquée 6 (1987) 383-407.

5. F. Fouchal, F. Lebon and I. Titeux, Contribution to the modelling of interfaces in masonry construction, Constr. Building Mater. 23 (2009) 2428-2441.

6. Z. Hashin, Thin interphase/imperfect interface in elasticity with application to coated fiber composites, J. Mech. Phys. Solids 50 (2002) 2509-2537.

7. I. V. Ivanov, D. S. Velchev, M. Knec and T. Sadowski, Computational models of laminated glass plate under transverse static loading, in Shell-line structures, Non-classical theories and applications, H. Altenbach, V. A. Eremeyev, eds. Advanced Structural Mathematics (Springer, Berlin, 2011), pp. 469-490.

8. S. Lenci, F. Clementi and J. Warminski, Nonlinear free dynamics of a two-layer composite beam with different boundary conditions, Meccanica 50 (2015) 675-688.

9. Y. D. Li, T. Xiong and L. Dong, A new interfacial imperfection coupling model (IICM) and its effect on the fracture behavior of a layered multiferroic composite: Anti-plane case, Eur. J. Mech.-A/Solids 52 (2015) 26-36.

10. J. C. Lopez-Realpozo, R. Rodriguez-Ramos, R. Guinovart-Diaz, J. Bravo-Castillero, J. A. Otero, F. J. Sabina, F. Lebon, S. Dumont and I. Sevostianov, Effective elastic shear stiffness of a periodic fibrous composite with non-uniform imperfect contact between the matrix and the fibers, Int. J. Solids Struct. 51 (2014) 1253-1263.

11. J. A. Nairn, Numerical implementation of imperfect interfaces, Comput. Mater. Sci. 40 (2007) 525-536.

12. C. Pelissou and F. Lebon, Asymptotic modeling of quasibrittle interfaces, Comp. Struct. 87 (2009) 1216-1223.

13. F. Fouchal, F. Lebon, M. L. Raffa and G. Vairo, An interface model including cracks and roughness applied to masonry, Open Civil Eng. J. 8 (2014) 263-271.

14. A. Rekik and F. Lebon, Identification of the representative crack length evolution in a multi-level interface model for quasi-brittle masonry, Int. J. Sol. Struct. 47 (2010) 30113021.

15. A. Rekik and F. Lebon, Homogenization methods for interface modeling in damaged masonry, Adv. Eng. Software 46 (2012) 35-42.

16. E. Bonetti, G. Bonfanti, F. Lebon and R. Rizzoni, A model of imperfect interface with damage, Meccanica 52 (2017) 1911-1922.

17. E. Bonetti, G. Bonfanti and R. Rossi, Global existence for a contact problem with adhesion, Math. Meth. Appl. Sci. 31 (2008) 1029-1064.

18. E. Bonetti, G. Bonfanti and R. Rossi, Well-posedness and long-time behaviour for a model of contact with adhesion, Indiana Univ. Math. J. 56 (2007) 2789-2819.

19. S. Dumont, F. Lebon and R. Rizzoni, An asymptotic approach to the adhesion of thin stiff films, Mech. Res. Comm. 58 (2014) 24-35.

20. G. Geymonat, F. Krasucki and S. Lenci, Mathematical analysis of a bonded joint with a soft thin adhesive, Math. Mech. Sol. 4 (1999) 201-225.

21. F. Lebon, A. Ould Khaoua and C. Licht, Numerical study of soft adhesively bonded joints in finite elasticity, Comput. Mech. Springer 21 (1998) 134-140.

22. A. Klarbring, Derivation of the adhesively bonded joints by the asymptotic expansion method, Inter. J. Eng. Sci. 2 (1991) 493-512.

23. R. Rizzoni and F. Lebon, Imperfect interfaces as asymptotic models of thin curved elastic adhesive interphases, Mech. Res. Comm. 51 (2013) 39-50.

24. R. Rizzoni, S. Dumont and F. Lebon, On Saint Venant - Kirchhoff imperfect interfaces, Inter. J. Non-Lin. Mech. 89 (2017) 101-115.

25. M. Serpilli, Asymptotic analysis of a multimaterial with a thin piezoelectric interphase, Meccanica 49 (2014) 1641-1652. 
26. I. Sevostianov and M. Kachanov, On some controversial issues in effective field approaches to the problem of the overall elastic properties, Mech. Mat. 69 (2014) 93-105.

27. I. Sevostianov, J. Kovik and F. Siman-k, Elastic and electric properties of closed-cell aluminum foams: Cross property connection, Mat. Sci. Eng. A $\mathbf{4 2 0}$ (2006) 87-99.

28. M. Frémond, Non-smooth Thermo-mechanics (Springer, Berlin, 2001).

29. A. Mielke, T. Roubíček and M. Thomas, From damage to delamination in nonlinearly elastic materials at small strains, J. of Elasticity 109 (2012) 235-273.

30. P. G. Ciarlet, Mathematical Elasticity Volume I: Three Dimensional Elasticity (NorthHolland, Amsterdam, 1988).

31. F. Lebon and R. Rizzoni, Asymptotic study on a soft thin layer the non-convex case, Mech. Adv. Mat. and Struct. 15 (2008) 12-20.

32. F. Lebon and R. Rizzoni, Asymptotic analysis of a thin interface: The case involving similar rigidity, Inter. J. Eng. Sci. 4 (2010) 473-486.

33. F. Lebon and R. Rizzoni, Asymptotic behavior of a hard thin linear elastic interphase: An energy approach, Inter. J. Solids Struct. 48 (2011) 441-449.

34. F. Lebon and R. Rizzoni, Modeling a hard, thin curvilinear interface, Disc. Cont. Dyn. Syst. Ser. S 6 (2013) 1569-1586.

35. F. Lebon, R. Rizzoni and S. Ronel-Idrissi, Asymptotic analysis of some non-linear soft thin layers, Comput. Strut. 82 (2004) 1929-1938.

36. F. Lebon and S. Zaittouni, Asymptotic modelling of interfaces taking contact conditions into account: Asymptotic expansions and numerical implementation, Inter. J. Eng. Sci. 48 (2010) 111-127.

37. R. Rizzoni, S. Dumont, F. Lebon and S. Sacco, Higher order model for soft and hard interfaces, Inter. J. Sol. Struct. 51 (2014) 4137-4148.

38. M. Kachanov, Elastic solids with many cracks and related problems, Adv. in Appl. Mech. 30 (1993) 259-445. 